\title{
Perspectiva de Género y Cooperativas No Agropecuarias en Cuba. Potenciales contribuciones desde la asesoría jurídica
}

\author{
Orestes Rodríguez Musa ${ }^{1}$ \\ Deibby Valle Ríos ${ }^{2}$ \\ Universidad de Pinar del Río, Cuba
}

Recibido: 26-11-2018

Aceptado: 21-12-2018

Sumario: I. Introducción. II. Perspectiva de Género y Cooperativas. Realidad de las Cooperativas no Agropecuarias en Pinar del Río. III. La asesoría jurídica de la cooperativa y su contribución a la identidad cooperativa. IV. Potenciales contribuciones desde la asesoría jurídica a la equidad de género en las Cooperativas No Agropecuarias de Pinar del Río. V. Conclusiones.

Resumen: El objetivo de este trabajo es fundamentar algunas propuestas destinadas a contribuir, desde la asesoría jurídica, con el fortalecimiento de la equidad de género en las Cooperativas No Agropecuarias de la provincia de Pinar del Río. Para ello primero se caracteriza, desde una perspectiva de género, la realidad de estas formas asociativas en la provincia. A continuación, se analiza teóricamente el proceso de asesoría jurídica, así como su contribución a la identidad cooperativa. Por último, se argumenta un conjunto de potenciales contribuciones desde el proceso de asesoría jurídica a la equidad de género en las Cooperativas No Agropecuarias del territorio.

Palabras claves: cooperativa; perspectiva de género; asesoría jurídica

Abstract: The objective of this work is to base some proposals destined to contribute, from the juridical advice, with the strengthening of the gender equality in the Urbans Cooperatives of the province of Pinar del Río. For this purpose, the reality of these associative forms in the province is characterized from a gender perspective. Next, the legal counseling process is analyzed theoretically, as well as its contribution to the cooperative identity. Finally, a set of

1 Profesor del Departamento. Facultad de Ciencias Sociales y Humanidades. Universidad de Pinar del Río. Cuba. E-mail: musa@upr.edu.cu

2 Licenciada en Psicología por la Universidad de la Habana, Cuba (2014). Profesora del Departamento de Gestión Sociocultural para el Desarrollo. Facultad de Ciencias Sociales y Humanidades. Universidad de Pinar del Río. Cuba. E-mail: deibby@upr.edu.cu 
potential contributions is argued from the process of legal advice to gender equity in the Urbans Cooperatives of the territory.

Keywords: cooperative; gender perspective; legal assistance 


\section{Introducción}

Los debates sobre "género» a nivel internacional resultan cada vez más generalizados, profundos y complejos. Esta categoría, al ser un producto resultante de los procesos histórico-culturales, muestra que las agudas diferencias entre mujeres y hombres, entre lo «femenino» y lo «masculino», no es posible enfocarlas desde las superadas posturas biologisistas, pues trascienden a todos los planos de la realidad social. Por ello, casi como un sello de modernidad, los estudios actuales (de prácticamente todo), cada vez más asumen la «perspectiva de género» como imprescindible para conseguir enfoques y resultados equitativos/transformadores.

Esta última idea también explica que paulatinamente políticas, programas y estructuras - públicas y privadas; locales, nacionales e internacionales - hayan venido contribuyendo (no con pocos obstáculos y múltiples retos) a remediar estas inequidades. Para ello, el Derecho no solo es una herramienta de implementación y de transformación social.

Entretanto, un espacio natural para aplicar estos enfoques de género lo constituyen las cooperativas, pues a su historia popular, a su concepción más pura y a sus mejores prácticas, le son consustanciales conocidos valores y principios que lo fundamentan.

Cuba, a partir del proceso de actualización de su modelo socioeconómico, ha implementado nuevas formas de trabajo cooperado en sectores diferentes al agropecuario. En esta Ilamadas Cooperativas No Agropecuarias (CNA), se ha promovido generalmente la igualdad entre el colectivo de socios trabajadores. Sin embargo, no se conocen relevantes esfuerzos destinados a fortalecer la equidad de género al interior de estas nuevas formas asociativas. Este asunto ha sido estudiado en los últimos años desde la Universidad de Pinar del Río.

La asesoría jurídica, como modo de actuación del profesional del Derecho, mediante un óptimo aprovechamiento de la autonomía cooperativa puede contribuir — de múltiples formas - a fortalecer la equidad de género en las cooperativas. Este proceso ha estado marcado por varias limitaciones en las CNA de Pinar del Río, pese a que en la provincia existen un conjunto de condiciones objetivas y subjetivas que deben ser aprovechadas en pos de transformar esta realidad.

En esta última dirección trabaja el Departamento de Derecho de la Universidad del territorio, de conjunto con la Empresa Provincial de Servicios Legales (EPSEL), a fin de perfeccionar la metodología seguida para desarrollar la asesoría jurídica a las CNA que funcionan en Pinar del Río.

Teniendo en cuenta que no es posible cumplimentar suficientemente este propósito sin transversalizarlo con una adecuada perspec- 
tiva de género, el presente trabajo tiene por objetivo fundamentar algunas propuestas para contribuir, desde la asesoría jurídica, con el fortalecimiento de la equidad de género en las CNA de la provincia de Pinar del Río. Para ello, inicialmente se caracteriza, desde una perspectiva de género, la realidad de estas formas asociativas en la provincia. A continuación, se analiza teóricamente el proceso de asesoría jurídica, así como su contribución a la identidad cooperativa. Por último, se argumenta un conjunto de potenciales contribuciones desde el proceso de asesoría jurídica a la equidad de género en las CNA del territorio.

\section{Perspectiva de Género y Cooperativas. Realidad de las Cooperativas no Agropecuarias en Pinar del Río}

El género, desde el punto de vista psicológico, se refiere al «conjunto de creencias compartidas por un grupo social sobre características psicosociales, es decir, rasgos, roles, motivaciones y conductas que se consideran propias de mujeres y hombres (género determinado)» ${ }^{3}$. Estas creencias se determinan en mayor grado por la cultura, la cual incide en los seres humanos a través del género, y este a su vez pauta las bases para el resto de otros procesos como la política, las religiones y la vida cotidiana en general. Vemos entonces, como el género se relaciona con el sexo, pero no lo agota 4 .

De igual forma, esta categoría funciona como un principio básico de organización de las sociedades, pues históricamente ha dividido el trabajo en espacios como la familia, las comunidades y el mercado. Las divisiones laborales mediatizadas por el género han marcado límites entre los hombres y las mujeres, siendo comúnmente las mujeres las más desfavorecidas. A ellas se les han asignado responsabilidades relacionadas con su rol reproductivo, que implican la crianza de los hijos y el cuidado de la familia. Además, aunque se involucren junto a los hombres en las actividades productivas, las funciones que desempeñan difieren en la mayoría de los casos de las de ellos ${ }^{5}$.

Así, el género plantea desafíos tales como los de particularizar y explorar las realidades más que asumirlas y reproducirlas. Esto nos permite no solo conocer los cambios en las relaciones que acontecen entre

3 VASALLO, N.: El género: Un análisis de la «naturalización» de las desigualdades. Editorial CEDEM, La Habana, 2004, pág. 141-152.

4 Ibidem.

5 CEDPA: «Género y Desarrollo: Serie de Manuales de Capacitación de CEDPA». CEDPA Volumen III, 1996 pág. 6. Recuperado de http://www.cedpa.org/images/pubspdfs/gender_spanish_all.pdf, el 5 de diciembre de 2018. 
hombres y mujeres, sino que además da pasos hacia la transformación de esas relaciones, dando un papel más activo a las féminas, separándolas de la subordinación histórica a la que han sido sometidas.

Para lograr estos cambios, la sociedad se ha apoyado en investigaciones con una perspectiva de género que ha contribuido a describir cómo surgen y se desarrollan las diferencias en las prácticas, discursos y representaciones sociales. A la vez, la perspectiva de género devela los esfuerzos destinados a atender las necesidades y prioridades específicas de los hombres y las mujeres, ya sea en conjunto o por separado. Estas nuevas herramientas se desarrollan en políticas, programas y estructuras institucionales que remedian desigualdades, así como custodian la preservación de derechos para ambos sexos.

En este sentido, la Oficina Internacional del Trabajo (OIT) alertó, en su «Política sobre la incorporación de la perspectiva de género» que:

«La incorporación de la perspectiva de género no consiste simplemente en añadir un «componente femenino» ni un «componente de igualdad entre los géneros» a una actividad existente. Es asimismo algo más que aumentar la participación de las mujeres. Significa incorporar la experiencia, el conocimiento y los intereses de las mujeres y de los hombres para sacar adelante el programa de desarrollo (...). El objetivo de la incorporación de la perspectiva de género es, por lo tanto, transformar las estructuras sociales e institucionales desiguales en estructuras iguales y justas para los hombres y las mujeres» ${ }^{6}$.

Un elemento esencial para lograr incorporar adecuadamente la perspectiva de género en centros de trabajo, lo constituyen en la actualidad la implementación de buenas prácticas laborales de equidad de género. El uso de estas buenas prácticas permite el diseño y aplicación de políticas, medidas y/o iniciativas que se suman a las normas laborales de las empresas de forma voluntaria, y son utilizadas para promover la equidad de género7, la igualdad de oportunidades entre trabajado-

6 Organización Internacional del Trabajo (OIT): Política de la OIT sobre la incorporación de la perspectiva de género, 1999. Recuperado de http://www.ilo.org/public/spanish/ bureau/gender/newsite2002/about/defin.htm, el 5 de diciembre de 2018.

7 «La equidad de género tiene que ver con la diversidad y el reconocimiento de las desigualdades, de la inequidad en las posibilidades de los seres humanos de acceder a las oportunidades para mejorar sus vidas. Del trato diferenciado pero equitativo que hay que dar a las personas, de acuerdo con su situación y condición de desventaja o desigualdad, para el logro de iguales oportunidades y el ejercicio pleno de los derechos en iguales condiciones». MÉnDEz. N.: «Estrategia de impulso al modelo cooperativo entre mujeres». Comité Regional de Equidad de Género (CREG), ACI Américas, 2011. pág. 9. Recuperado de www. aciamericas.coop/IMG/pdf/estrategiamodelocoopmujeres.pdf, el 5 de diciembre de 2018. 
ras y trabajadores. A partir de su ejecución, se ha constatado que, por lo general, se logran eliminar las brechas existentes.

Esta alternativa es favorable para las empresas, pues permite que en ellas se establezcan óptimos niveles de calidad y gestión. Dentro de los beneficios de realizar buenas prácticas laborales de equidad de género destacan la obtención de mayor flexibilidad para enfrentar los cambios, aumento de las opciones en la retención de empleados, incremento de la productividad y efectividad del trabajo, disminución de los índices de ausentismo, mejor clima laboral, obtención de una imagen pública más atractiva para la fuerza laboral y mayor compromiso con la organización ${ }^{8}$.

Si tenemos en cuenta que - históricamente- entre los objetivos de la cooperativa se halla la creación de estructuras empresariales que garanticen la viabilidad económica junto a la justicia social, la democracia y el reparto de poder ${ }^{9}$, cabe asegurar que pueden resultar espacios ideales para la implementación de buenas prácticas laborales desde la perspectiva en cuestión.

La Alianza Cooperativa Internacional $(\mathrm{ACl})$, a través del principio de «asociación voluntaria y abierta» orienta las pautas a seguir para alcanzar la equidad entre socias y socios, sin discriminaciones raciales, políticas, religiosas, sociales y de género ${ }^{10}$. Por tanto, estas instituciones están llamadas a crear, conforme a su naturaleza, las condiciones necesarias para que todos y todas expresen y defiendan libremente sus opiniones, sin que en este proceso medie una relación de poder.

La propia $\mathrm{ACl}$, en el año 2000, aporta dos razones por las que las cooperativas deben fomentar la igualdad de género: «Por un lado, las razones morales y éticas basadas en los derechos humanos básicos de hombres y mujeres; por otro lado, la promoción de un crecimiento económico basado en la total utilización de los recursos humanos»» ${ }^{11}$.

8 SERNAM, Acción RSE: Guía para impulsar la Equidad de Género en las empresas. 2006. Recuperado de http://www.centroscomunitariosdeaprendizaje.org.mx/sites/default/files/guia_para_impulsar_la_equidad.pdf el 5 de diciembre de 2018.

9 Cemborain, E.: Responsabilidad social en las cooperativas: igualdad de oportunidades entre hombres y mujeres, 2006. Recuperado de www.researchgate.net/ publication/268299116_Responsabilidad_social_en_las_cooperativas_igualdad_de_ oportunidades_entre_hombres_y_mujeres, el 5 de diciembre de 2018.

10 Alianza Cooperativa Internacional: Declaración Sobre la Identidad Cooperativa, Manchester, 1995. Recuperado de http://www.elhogarobrero1905.org.ar, consultada el 19 de diciembre de 2009.

11 Alianza Cooperativa Internacional (ACl): Estrategia para promover la igualdad de género, 2000. Recuperado de http://www. coop.org/coop/2000-gender-strategy.pdf, el 5 de diciembre de 2018. 
Tal cual apuntan Esteban, Gargallo \& Pérez, «lograr representatividad de género en los Consejos de Administración de estas empresas conllevaría mayor efectividad en su funcionamiento. Las mujeres, por ejemplo, facilitarían la obtención de resultados de gobierno corporativo en la cooperativa, pues juegan roles directos como líderes, mentoras o miembros de redes, pero también ejercerían influencias indirectas convirtiéndose en símbolos al alcanzar las metas que se proponen, ofreciendo nuevas opciones a otras mujeres y siendo fuente de inspiración para estas ${ }^{12}$.

Pese a las oportunidades que aquí se generan para lograr una adecuada perspectiva de género, aun se corre el riesgo de que los modos habituales de discriminación sean sufridos como producto generalizado en la sociedad y vistos entonces de forma natural. Esto puede incidir en que, el funcionamiento interno y externo de la cooperativa reproduzcan conductas, tradiciones y costumbres estereotipadas contrarias al carácter integrador e inclusivo de estas empresas.

Tomando como base este acercamiento teórico a la perspectiva de género en las cooperativas, este trabajo se adentra en la realidad cubana, donde la actualización del modelo socioeconómico nacional ha impulsado un proceso experimental con un grupo de CNA surgidas a mediados del año $2013^{13}$

Desde sus inicios, en las CNA se ha promovido la igualdad entre el colectivo de socios-trabajadores desde la legislación general y desde los Estatutos; sin embargo, no se conocen relevantes esfuerzos dedicados a consolidar dicha igualdad desde una perspectiva de género al interior de estas nuevas formas asociativas.

La provincia de Pinar del Río, respondiendo a lo que ya parece una tradición histórica ${ }^{14}$, fue una de las primeras del país en materializar la

12 Esteban, M.L., Gargallo, A. \& Pérez, F.J.: «¿Presentan las cooperativas contextos favorables para la igualdad de género? Especial referencia a la provincia de Teruel». CIRIEC-España, Revista de Economía Pública, Social y Cooperativa, 88, 2016, pág. 68.

13 Para más detalles sobre este proceso de expansión de las cooperativas hacia otros sectores de la economía nacional diferentes al agropecuario, vid. RODRíGUEZ, O.: La constitucionalización de la cooperativa. Una propuesta para su redimensionamiento en Cuba. Ed. Vincere Asociados, Coletânea IBECOOP (No. 1), Brasilia-DF, 2017, pág. 107-129.

14 El movimiento de la agricultura comenzó por la provincia de Pinar del Río, donde inicialmente se agruparon en 87 cooperativas a más de 10 mil campesinos individuales, por lo general beneficiados por las leyes de reforma agraria. Vid. CASTRO, F.: Discurso de Clausura del Primer Congreso Provincial Campesino en Santiago de Cuba el 24 de febrero de 1959, en La Reforma Agraria. Obra Magna de la Revolución, Oficina del Historiador de la Ciudad de la Habana, La Habana, 2006; referido en NovA, A.: «Las cooperativas agropecuarias en Cuba: 1959-presente» en PINEEIRO, C. (compiladora): Cooperativismo y Socialismo. Una mirada desde Cuba. Ed. Caminos, La Habana, 2012, pág. 322. 
expansión de las cooperativas hacia otros sectores de la economía diferentes al agropecuario. Además, es reconocida entre la comunidad científica del país, la trayectoria investigativa acumulada de la Universidad del territorio sobre los temas cooperativos.

Todo lo anterior justifica que, en esta investigación con perspectiva de género, se haya prestado especial atención a los resultados de investigación desde allí emergidos con anterioridad. Entre estos resultados destacan (por recientes), los estudios de casos realizados por un grupo de estudiantes del 4to. Año de la Carrera de Derecho durante el Curso Académico 2016-2017 como parte de su evaluación final en la Asignatura Optativa de Introducción al Derecho Cooperativo. En base a estas y otras $^{15}$ fuentes, se sistematizan a continuación algunas de las limitaciones constatadas desde la práctica, relacionadas con la perspectiva de género.

En un primer análisis vale destacar, que la redacción de las normas (generales e internas) que rigen estas cooperativas resulta tradicional, en tanto predomina un lenguaje sexista que solo alude a los «socios». La exclusión de las socias en este decir del hacedor de las normas contribuye a invisibilizar a las mujeres 0 , cuando menos, a generar una imagen secundaria de ellas, reforzando las desigualdades e inequidades respecto a los hombres.

Al respeto destaca el bajo nivel de escolaridad de las socias, que en todos los casos oscilan entre el Medio-Básico (9no. grado) y el MedioSuperior (12mo. grado), factor que incide - negativamente - en la influencia que podrían ejercer las féminas durante el proceso de toma de decisiones colectivas, así como en sus posibilidades de ocupar puestos de mayor trascendencia y responsabilidad.

En este sentido, es pertinente apuntar que los cargos de dirección son ocupados en su mayoría por hombres, siendo estos los que desem-

15 Vid. HeRnÁndez, O.: «La relación género y cooperativa en el ordenamiento jurídico cubano. Una aproximación a su realidad y a sus perspectivas ante el proceso de perfeccionamiento del modelo económico en el país». Boletín de la Asociación Internacional de Derecho Cooperativo, Núm. 46, 2012, pág. 297-320; HeRnÁndez, O.: "La relación género y cooperativa en Cuba. Perspectivas dentro del marco jurídico experimental de la cooperativa». Revista de Estudios Cooperativos, N. 2, 2013, pág. 133-156; HeRNÁNDEZ, O.: "Las cooperativas no agropecuarias en Pinar del Río. Una aproximación al enfoque de género en su funcionamiento». Revista de Estudios Cooperativos. Núm. 6, 2015, pág. 159-179; HeRNÁNDEZ, O.: «La relación entre enfoque de género y trabajo en las Cooperativas No Agropecuarias en Pinar del Río. Reflexiones para su perfeccionamiento.» Ponencia presentada en X Encuentro de Investigadores Latinoamericanos en Cooperativismo, Buenos Aires, Argentina, 2018; Hernández, O., Valle, D.: La Cooperativa No Agropecuaria Atelier "La Moda»: Aproximación a la experiencia de una cooperativa de mujeres en Rodríguez, O. y Hernández, O. (compiladores): «Apuntes de Derecho Cooperativo para Cuba». Ediciones Loynaz, Pinar del Río, 2018, págs. 264-288. 
peñan funciones como las de Presidente, Sustituto del Presidente o Administrador; y quedan para las socias puestos como el de Secretaria. En tales asignaciones se reproducen estereotipos de género, permeados por la segregación vertical ${ }^{16}$ y horizontal ${ }^{17}$.

Por otra parte, las condiciones de trabajo son irregulares y con ellas mayormente se sienten más a gusto los socios de las cooperativas, mientras que las socias hacen énfasis en los problemas e insuficiencias de los locales y áreas en las que se desempeñan como trabajadoras. Estas condiciones influyen en el bajo nivel de satisfacción de las socias con sus condiciones de trabajo.

A lo anterior se suma que, ante el aumento de la demanda de trabajo en algunas cooperativas, no es común que se incorpore nueva fuerza de trabajo, sino que los socios y socias optan por multiplicar sus esfuerzos. Este incremento de la carga redunda en un aumento especialmente significativo para las mujeres, que al llegar a casa deben enfrentarse a las labores domésticas que conforme a los estereotipos de género tradicionalmente le han venido asignadas.

En otra dirección resalta la falta de expresa referencia, entre los principios declarados por la legislación general de las CNA, a la «Educación, Información y Capacitación». Esta preterición del legislador cubano sobre la «regla de oro del cooperativismo», incide en la ausencia de un andamiaje institucional interno que asegure su realización, que a su vez redunda específicamente en la capacitación sobre temáticas con perspectiva de género ${ }^{18}$.

16 «Es la disparidad presencial en las jerarquías institucionales: las mujeres constituyen mayoría en los puestos de menor remuneración, poco valorados y con menos poder de decisión». Vid. CuADRADO, I.: Valores y rasgos estereotípicos de género en mujeres líderes. En Revista Psicothema, Vol. 16, No. 2, 2004, págs. 270-275.

17 «La disparidad se concentra en áreas laborales, o a saber «feminizadas» o «masculinizadas»: en las universidades, aunque la mayoría de la matrícula esté compuesta por mujeres, estas se concentran en carreras de ciencias sociales, naturales y humanísticas; y los hombres en las de ciencias exactas, técnicas y en las ingenierías. En el campo empresarial, las mujeres son mayoría en áreas de servicios; los hombres, en técnicas y de producción». Vid. MunduATE, L.: Género y liderazgo. Diferencias entre hombres y mujeres en el acceso a puestos directivos. Revista de Psicología Social, Vol. 18, Núm. 3, 2003; BARBERÁ, E.: Género y organización laboral: intervenciones y cambio. En, FernánDEZ, J., (Coord.): La intervención en los ámbitos de la sexología y la generología. Madrid: Pirámide, 1999, pp. 22-31; referido en VIRGILI. D.: "Género y trabajo remunerado. Experiencia grupal de investigación-acción con mujeres y hombres dirigentes del Ministerio de Transporte». Tesis en opción al título de Máster en Psicología Educativa. Universidad de la Habana. Facultad de Psicología, 2012, págs. 11-13.

18 PIÑEIRO. C.: Diagnóstico preliminar de las cooperativas no agropecuarias en La Habana, Cuba, 2014. Recuperado de https://www.ceec.uh.cu/file/569/download?token=VfqEKMZE, el 18 de abril de 2016. 
Dichas carencias en el área de la educación, tienen además un importante efecto negativo en el contenido de los Estatutos y otras normas internas. Estos Estatutos no reflejan hoy una perspectiva de género consolidada, en tanto no se encuentran pronunciamientos sobre temas tan sensibles como la licencia de maternidad y paternidad; la flexibilidad de los horarios laborales en pos de la conciliación ${ }^{19}$ de mujeres y hombres con la vida societaria, laboral, familiar y personal, etc.

A tono con este problema, es criticable que la evaluación oficial del experimento ha sido fundamentalmente económica y no social, porque se ha entendido a la cooperativa estrictamente como un "actor económico» 20 , al que se le da «seguimiento económicamente», minimizándose la evaluación de lo que socialmente puede y debe aportar a la comunidad. De ahí que generalmente no se logre aún en la provincia la necesaria articulación de las CNA con las familias de los socios/socias, sus espacios domésticos y las comunidades que las acogen.

Por tanto, la realidad de la CNA en Cuba muestra que aún distan de convertirse en el espacio idóneo para lograr equidad entre socios y socias. De cara a estas limitaciones, se analizará a continuación el papel de la asesoría jurídica y su contribución a la identidad cooperativa.

\section{La asesoría jurídica de la cooperativa y su contribución a la identidad cooperativa}

La asesoría jurídica es un modo de actuación del profesional del Derecho sobre la cual no abundan referentes teóricos, quizás porque se ha subestimado, entendiéndola como una mera labor accesoria de poca incidencia en cuestiones jurídicas esenciales, algo que está lejos de la realidad.

19 Según Senent, en España la Ley Orgánica Núm. 3 de 22 de marzo de 2007, «presta especial atención a la conciliación en el ámbito específico de las relaciones laborales, mediante su reconocimiento expreso como derecho (art. 44), y con la incorporación de una serie de previsiones con las que se procura fomentar una mayor corresponsabilidad entre mujeres y hombres en la asunción de obligaciones familiares». Senent, M.: ¿Cómo pueden aprovechar las cooperativas el talento de las mujeres? Responsabilidad Social Empresarial e Igualdad Real, Revesco N. 105 - Monográfico: La economía social y la igualdad de género, 2011, pág. 77.

20 Según TRISTÁ las CNA reportan mensualmente su estado económico y financiero a la Oficina Nacional de Estadísticas. TRISTÁ, G.: Cooperativas no Agropecuarias, conferencia ofrecida y reproducida a militantes del Partido Comunista de Cuba por la Comisión de Implementación de los Lineamientos del Partido y la Revolución, 2015. 
El asesor jurídico ${ }^{21}$ regularmente ejecuta un conjunto de funciones que pueden variar atendiendo al tipo de persona destinataria de sus servicios (física o colectiva) y de las normas que en cada país regulen este asesoramiento. No obstante, el enfoque de este trabajo será hacia la asesoría jurídica de la empresa cooperativa.

Sobre esta base, es pertinente ofrecer una sistematización de aquellas actividades fundamentales que desarrolla el asesor jurídico para una empresa22, a saber:

- Diagnosticar: implica la determinación del estado de cualquier fenómeno. Los resultados conseguidos deben funcionar como punto de partida para trazar y ejecutar el plan de acciones destinado a eliminar las dificultades jurídicas detectadas.

- Orientar: se manifiesta cuando el asesor aconseja a la empresa sobre las opciones y medios jurídicos con que se cuenta para satisfacer sus intereses, esclareciendo dudas y advirtiendo sobre el alcance y posibles efectos jurídicos de las acciones a emprender relativas a sus asuntos.

- Redactar: requiere de la «ingeniería» o creatividad del asesor, para reflejar principios e intereses concretos en documentos de trascendencia jurídica, especialmente cuando escribe la normativa interna (acuerdos, actas, reglamentos, y otras normas relativa a las funciones de la gerencia o aplicables a los trabajadores, por ejemplo contratos para formalizar vínculos con trabajadores y otras empresas); los documentos comprobatorios de las operaciones internas (órdenes de pago, letras de cambio y pagarés); y los poderes de actuación que otorga la empresa para su participación en actuaciones judiciales.

- Representar: se configura cuando una empresa (cliente), manifiesta su voluntad para que el asesor jurídico ejecute las acciones legales pertinentes que le permitan brindar solución a la si-

21 Para desempeñarse como asesor jurídico es necesario «ser profesional del Derecho, egresado de una Universidad reconocida con el título que acredite tal condición, regularmente matriculado en el gremio profesional correspondiente y con las habilitaciones legales requeridas» Vid. GARCíA, A: Derecho cooperativo y de la Economía Social y Solidaria, Asociación Iberoamericana de Derecho Cooperativo, Mutual y de la Economía Social y Solidaria, Mérida, 2017, pág. 67.

22 Vid. BRUCH, E.: Marketing de servicios jurídicos a pequeñas y medianas empresas. Universitat Autónoma de Barcelona, 2016, págs. 40-44. Recuperado de https://www.tesisenred.net/bitstream/handle/10803/386530/ebm1de1.pdf, el 24 de junio de 2018; La transformación de la función jurídica. Los siete retos de las asesorías jurídicas internas, s/f. Recuperado de https://www.pwc.es/es/publicaciones/legal-fiscal/assets/informe-pwc-transformacion-funcion-juridica.pdf, el 24 de junio de 2018 y GARCíA, A: ob. cit, pág. 25-30. 
tuación jurídica en que se encuentra, actuando en interés y por cuenta de esta.

- Supervisar: se materializa fundamentalmente al realizar la vigilancia en favor de la legalidad de los actos o procedimientos desarrollados, tales como la aplicación de medidas disciplinarias; el cumplimiento de las formalidades de los contratos concertados; la ejecución de estos contratos para la adquisición o suministro de bienes y servicios; el respeto a las obligaciones tributarias; etc.

Vale aclarar, que si bien a efectos metodológicos conviene delimitar estas actividades fundamentales, la práctica demuestra que ellas pueden manifestarse indistintamente en cualquier momento del proceso de asesoría jurídica. Por tanto, durante este proceso deben articularse, de forma armónica, las funciones de diagnóstico, consejería, ejecución y control que en su desempeño profesional desarrolla el asesor jurídico, a fin de equilibrar los intereses del cliente (empresa) y el interés general expresado en las exigencias legales.

Ahora bien, cuando hablamos de la asesoría jurídica de las cooperativas, debe tenerse en cuenta que estas poseen particularidades que las diferencian de otras empresas. Esta identidad propia de las cooperativas, impone a los asesores jurídicos que asuman compromisos en este campo, además de un determinado nivel de especialización. Del cumplimiento de este cuasi requisito depende —en importante medidaque la figura se manifieste conforme a su naturaleza.

Los elementos que ofrecen contenido a la identidad cooperativa se han abordado en otras ocasiones de cara a la labor constituyente/legislativa ${ }^{23}$. Ahora vale reiterar, desde este nuevo enfoque, que el asesor jurídico debe reconocer en la cooperativa un fenómeno socioeconómico integral, que trasciende el mero espacio empresarial y se complejiza sanamente.

Una cooperativa es una empresa, y como tal precisa de rentabilidad económica, pero en ella también toman forma jurídica vínculos asociativos inspirados en valores nacidos de necesidades sociales; a la vez que se superponen, sin conflictos, roles tradicionalmente enfrentados como el de propietario y trabajador, o el de empresario y consumidor. Además, la finalidad de servicio a los asociados que caracteriza a la cooperativa, cuando menos trastoca la perspectiva tradicional del lucro, también porque la cooperativa no tiene en la responsabilidad social un apéndice impuesto, como sucede en otras tantas oca- 
siones, sino porque es inherente a su naturaleza y estructura institucional. En el mismo sentido, no se debe olvidar que el funcionamiento de esta empresa se asienta en principios como la voluntariedad, la igualdad, el control democrático, la distribución equitativa, la autonomía y la educación, que comúnmente no pasan de ser aspiraciones o - peor aún- un eslogan de muchos entes públicos y privados.

Por consiguiente, a los actos jurídicos fundamentales que establecen las cooperativas con sus socios o con otras cooperativas 24 , no le son aplicables — sin distorsionarlas - las normas que informan a ramas del Derecho como el Civil, el Mercantil o el Laboral. La naturaleza jurídica sui géneris de la cooperativa exige la aplicación a ella de una pujante y particular rama del Derecho.

El maestro HENRŸ apela a una dimensión amplia de este Derecho, al definirle como "todas aquellas normas jurídicas -leyes, actos administrativos, resoluciones judiciales, jurisprudencia, reglamentos y estatutos cooperativos y cualquier otra fuente de Derecho- que regulan la estructura y las operaciones de las cooperativas como empresas en el sentido económico y como entidades en el sentido jurídico» ${ }^{25}$.

Por su parte, la Ley Marco para las Cooperativas de América Latina, en un intento de unificación del Derecho Cooperativo a nivel regional, ofrece en su artículo 6 una definición de este, entendiéndolo como «el conjunto de normas especiales, jurisprudencia, doctrina y práctica basadas en los principios que determinan y regulan la actuación de las cooperativas y los sujetos que en ellas participan ${ }^{26}$.

Sin embargo, vale la advertencia sobre dos tendencias contradictorias: «por un lado, existe un respeto creciente por el derecho internacional público cooperativo y por la obligación de mantener a las cooperativas como una entidad jurídica diferenciada y, por otro lado, continúa el proceso de alineación o equiparación de las cooperativas

24 Explica CRACogna las notas esenciales y consustanciales a estos actos que permiten afirmar que no tienen una naturaleza civil ni comercial ni otra cualquiera, sino una que les es propia y que los distingue dada la propia finalidad de la institución: a) intervención de socio y cooperativa; b) objeto del acto idéntico al objeto de la cooperativa; y c) espíritu de servicio, donde hay un corpus (el objeto material o inmaterial sobre el que versa) y un animus (el espíritu de servicio que informa la relación). CRACOGNA, D.: Estudios de Derecho Cooperativo, Intercoop Ed. Cooperativa Ltda., Buenos Aires, 1986, pág. 21. También en este sentido SALINAS, A.: Derecho Cooperativo, Ed. Cooperativismo, México, 1954, pág. 2.

25 HENRŸ, H.: Orientaciones para la legislación cooperativa, OIT, Ginebra, 2013, pág. 11.

26 ACl: Ley Marco para las Cooperativas de América Latina, Ed. ACl-Américas, San José, 2009. 
con las sociedades comerciales» ${ }^{27}$. Además, «la convergencia del Derecho Cooperativo con el de las sociedades de capital (...) también puede ser vista como la aplicación - a veces, indiscriminada- a las cooperativas de normas que fueron diseñadas para las sociedades de capital, pero que en los hechos terminan dando forma a las cooperativas como instituciones $y / 0$ definiendo sus operaciones» ${ }^{28}$.

En el mismo sentido es de resaltar que "...los legisladores incluyen cada vez menos normas imperativas (ius cogens) dentro del Derecho Cooperativo. Considerando las presiones de los mercados financieros, los cooperativistas pueden aprovechar el reducido alcance de la legislación vinculante y elaborar estatutos que abran el camino para la alineación de las cooperativas con las sociedades comerciales» o, por el contrario, pueden usar esta flexibilidad para reforzar su identidad. No en balde resaltan, durante los últimos años, los debates teórico/prácticos sobre la aplicabilidad a las cooperativas de las recomendaciones contenidas en los Códigos de Buen Gobierno Corporativos ${ }^{29}$.

En todo caso, debemos tener en cuenta que «la gestión social y «societal» de las cooperativas a través de profesionales independientes y calificados, junto con el asesoramiento sobre cómo mejorar la gestión y administración, es una condición sine qua non para un desarrollo saludable de las cooperativas» ${ }^{30}$. Por tal motivo, el papel del asesor jurídico es definitorio en los derroteros que asumen estas formas asociativas.

Por tanto, esta potencial incidencia de la asesoría jurídica es proporcional al nivel de institucionalización con que cuente el Derecho Cooperativo en cada ordenamiento jurídico; pero también es determinante en este sentido la postura crítica y conciencia creativa que despliegue el asesor jurídico durante el desarrollo de sus funciones de diagnóstico, consejería, ejecución y control, al redactar, interpretar y aplicar las normas.

27 HeNRŸ, H.: ob. cit, pág. 12.

28 Ibidem, pág. 13.

29 Vid. Cracogna, D. y UriBe, C.: Buen Gobierno Cooperativo. VIII Congreso Nacional Cooperativo, Cartagena de Indias, 2003. Recuperado de http://www.confecoop.coop/images/stories/memorias/2003/dante_uribe\%20_\%28Buen\%20Gobierno\%, el 5 de diciembre de 2018 y Lineamientos frente al Código de Buen Gobierno para el Sector Solidario-Cooperativas. Manual Guía Gobierno Corporativo Cooperativas, EMPRENDER, 2017. Recuperado de https://www.google.com/url?sa=t\&rct=j\&q=\&esrc=s\&source=web\&cd=2\&ved=2ah UKEwjE-o_NIKbfAhXNmVkKHS9xC5gQFjABegQIARAC\&url=http\%3A\%2F\%2Fwww.campusvirtualemprender.com\%2Fmoodle\%2Fpluginfile.php\%2F2555\%2Fblock_html\%2Fcontent\%2FManual\%2520Gui\%25CC\%2581a\%2520Gobierno\%2520Corporativo\%2520C ooperativas.pdf\&usg=AOvVaw0jpF7d2yVeKqQbxO4_jJ6O, el 5 de diciembre de 2018.

30 HeNRŸ, H.: ob. cit, pág. 107. 


\section{Potenciales contribuciones desde la asesoría jurídica a la equidad de género en las Cooperativas No Agropecuarias de Pinar del Río}

Recientemente se ha reconocido oficialmente en Cuba que algunas cooperativas (no agropecuarias) actúan como empresas privadas, donde el presidente ejerce como si fuera el dueño, con un mínimo de socios, a la vez que realizan su gestión fundamentalmente contratando los servicios de trabajadores por cuenta propia como asalariados, para luego repartir las utilidades solo entre los socios ${ }^{31}$.

Teniendo en cuenta estas y otras dificultades, se ha decidido por la dirección del país, "antes de continuar avanzando en la creación de nuevas cooperativas, consolidar lo avanzado, generalizar los aspectos positivos, que no son pocos, y enfrentar resueltamente las ilegalidades y otras desviaciones que se apartan de la política establecida» ${ }^{32}$

Esta directriz ha derivado en hacer más efectivo el control y la fiscalización desde el Estado sobre las cooperativas ${ }^{33}$, así como en la reciente decisión administrativa de extinguir algunas de las nuevas cooperativas que se habían autorizado ${ }^{34}$. Sin embargo - a consideración de los autores - esta es una estrategia con «piernas cortas», que deberá ser sustituida por proyectos de acompañamiento que tengan como brújula la identidad cooperativa universalmente reconocida.

Concretamente, respecto a la asesoría jurídica en Cuba, recién ha entrado en vigor el Decreto Ley 349 de 24 de enero de 2018, «Del Asesoramiento Jurídico» y la Resolución 41 de 3 de marzo de 2018 del

31 Vid. PUIG, Y.: Autoridades explican nuevas medidas respecto a cooperativas no agropecuarias, 2017. Recuperado de http://www.cubadebate.cu/noticias/2017/08/09/ autoridades-explican-nuevas-medidas-respecto-a-cooperativas-no-agropecuarias/, el 12 de septiembre de 2017.

32 CAstro, R.: Discurso pronunciado por el General de Ejército, Primer Secretario del Comité Central del Partido Comunista de Cuba y Presidente de los Consejos de Estado y de Ministros, en la clausura del IX Período Ordinario de Sesiones de la VIII Legislatura de la Asamblea Nacional del Poder Popular, La Habana, 14 de julio de 2017, Versiones Taquigráficas del Consejo de Estado, Granma, Órgano Oficial del Comité Central del Partido, 15 de julio de 2017.

33 Vid. PUIG, Y.: ob. cit.

34 Vid. Cubadebate: Autorizadas 16 cooperativas no agropecuarias en primer trimestre de 2016, 2016, recuperado de http://www.cubadebate.cu/noticias/2016/06/29/ autorizadas-16-cooperativas-no-agropecuarias-en-primer-trimestre-de-2016/, el 4 de octubre de 2016; y MinISTERIO DE FINANZAS Y PRECIOS: Aprobada extinción de Cooperativa de servicios contables SCENIUS, 2017, recuperado en http://www.cubadebate.cu/ noticias/2017/08/07/aprobada-extincion-de-cooperativa-de-servicios-contables-scenius/\#.Wb54j3uR7mM, el 12 de septiembre de 2017. 
MINJUS «Reglamento para el ejercicio de la actividad de asesoramiento jurídico». Dichas normas, si bien contemplan a la CNA entre los sujetos destinatarios de este servicio ${ }^{35}$, no es su objeto revertir el panorama de escasa especialización en la materia cooperativa de que adolecen los juristas del país, ni tampoco fortalecer, desde el desempeño de estos profesionales, la identidad de las instituciones en cuestión.

Sin embargo, contrasta con esta realidad que el Departamento de Derecho de la Facultad de Ciencias Sociales y Humanidades de la Universidad de Pinar del Río (UPR), —casa de altos estudios que como antes se ha dicho ${ }^{36}$, cuenta con una importante trayectoria investigativa en estos temas - ejecutó entre el año 2013 y el 2016 un Proyecto de Investigación y Desarrollo que fundamentó una propuesta de "Bases teóricas para la efectiva expansión jurídica de las cooperativas hacia otras esferas de la economía nacional además de la agropecuaria».

Este Proyecto obtuvo importantes resultados, que se reflejan en una Tesis de Doctorado en Ciencias Jurídicas; 20 publicaciones en libros o revistas científicas; 33 presentaciones en eventos científicos, de ellas más del $80 \%$ internacionales; 1 Grupo Científico Estudiantil que vinculó sus Trabajos de Curso a los objetivos; 4 Tesis de Grado de Licenciatura en Derecho asociadas; 1 asignatura optativa impartida en la Carrera de Derecho de la UPR durante cuatro cursos; 1 evento internacional propio con proyecciones futuras; 2 ediciones de un curso de posgrado impartido a un total de 48 juristas de la provincia; 11 premios a los resultados, algunos de ellos de carácter nacional; y vínculos con instituciones internacionales de referencia en el campo, con disposición para continuar colaborando para avanzar en esta dirección.

En base a estos antecedentes, que muchas veces han implicado espacios de diálogo, se han identificado múltiples oportunidades para que los asesores jurídicos de las doce (12) CNA de la provincia de Pinar del Río, vinculados en su totalidad a la Empresa Provincial de Servicios Legales (EPSEL), perfeccionen el proceso de asesoría a esta particular forma de empresas, contribuyendo a potenciar o consolidar los rasgos que le identifican.

Por consiguiente, se formalizó en enero de este año un Convenio entre la EPSEL y la UPR, contentivo de un Proyecto de Investigación, Desarrollo e innovación, destinado a implementar una «Metodología para perfeccionar el proceso de asesoría jurídica de las Cooperativas No Agropecuarias en la provincia de Pinar del Río, desde su gestación

35 Vid. Decreto Ley 349 de 24 de enero de 2018, «Del Asesoramiento Jurídico», Gaceta Oficial No. 5 Extraordinaria de 24 de enero de 2018.

36 Vid. Supra, epígrafe II. 
hasta su disolución» que, de ofrecer buenos resultados, podría generalizarse hacia otros lugares del país.

En este Proyecto, que apenas viene concluyendo su etapa inicial de diagnóstico, se han definido múltiples variables hacia las cuales la nueva metodología habrá de dirigir sus recomendaciones, a fin de contribuir con la identidad cooperativa. Entre estas variables se encuentra «la perspectiva de género», entre todas quizás la más trasversal.

Para argumentar los elementos que podrían dar cuerpo a esta propuesta (requerida de debate y perfeccionamiento) y de cara a las CNA de la provincia de Pinar del Río, es oportuno observar las recomendaciones realizadas por $\mathrm{ACl}$-Américas mediante su Comité Regional de Equidad de Género $(\mathrm{CREG})^{37}$, en tanto constituyen líneas estratégicas cruciales que pueden funcionar como guía para igualar derechos y oportunidades en asociadas y asociados, a saber:

- Incluir a mujeres y hombres cooperativistas en los puestos de toma de decisiones de mayor poder en el sector, generando igualdad de derechos y oportunidades.

- Transversalizar las acciones en materia de igualdad y equidad de género en los presupuestos de las cooperativas.

- Asignar fondos específicos para el trabajo de incidencia de la equidad de género en las cooperativas.

- Capacitar en los temas de género y cooperativas, lo que incluye comprender el enfoque de la planificación del desarrollo en los planos políticos, económicos, sociales, culturales y ambientales, con el interés de formar en planificación de género y su uso en las políticas, planes y programas de las cooperativas.

- Sensibilizar a las instancias de toma de decisiones en el enfoque de equidad de género y la planificación del desarrollo, lo que incluye la formación de mujeres y hombres cooperativistas que ocupan los puestos de poder en el sector.

- Formar promotoras y promotores que multipliquen en las cooperativas, los temas de equidad de género, liderazgos, derechos humanos, educación cooperativa y los valores y principios cooperativos con equidad de género.

- Realizar intercambios de experiencias exitosas en materia de igualdad y equidad de género entre las cooperativas.

37 Méndez. N.: «Estrategia de impulso al modelo cooperativo entre mujeres». Comité Regional de Equidad de Género (CREG), ACI Américas, 2011. Recuperado de www.aciamericas.coop/IMG/pdf/estrategiamodelocoopmujeres.pdf, el 5 de diciembre de 2018. 
- Crear o fortalecer las instancias especializadas en materia de equidad de género, favoreciendo la participación de las mujeres en estos espacios.

- Formar o capacitar a quiénes se encargan de estos espacios, como mecanismo de seguimiento y monitoreo de la igualdad y equidad de género en las cooperativas.

- Elaborar artículos o documentos que den cuenta de las acciones que se desarrollan en las cooperativas, para el avance de la igualdad y equidad de género.

Vale resaltar la alineación de estas recomendaciones con las bondades de la auto-organización, la auto-regulación y el auto-control que debe prevalecer en las cooperativas como manifestaciones de la autonomía que les caracteriza. A fin de cuentas «la autonomía de las cooperativas es también autonomía de valores frente a la influencia de prácticas heredadas de una cultura patriarcal....»38. Conservar esa autonomía implica el compromiso de no reproducir acríticamente la inequidad de género en el funcionamiento de estas organizaciones. Este funcionamiento lo definen —en importante medida — las políticas internas y disposiciones jurídicas de que se dota a sí misma la cooperativa (Estatutos, Reglamento Interno, Acuerdos de la Asamblea, etc.), en cuya elaboración, interpretación y aplicación es protagonista el asesor jurídico.

En consecuencia, desde la ordenación interna que define la vida de la CNA puede flexibilizarse la gestión del tiempo de trabajo (reordenación de horarios, posibilidad de acumular horas, evitar reuniones fuera del horario normalizado, consideración del calendario escolar, etc.) y del espacio laboral (fomento de las labores desde el domicilio, trabajo por objetivos o compartido, etc.), así como facilitar permisos, reducciones de jornadas, etc. De forma similar, para mitigar las sobrecargas que generalmente recaen sobre las féminas, pueden acordarse medidas o políticas en pos de asegurar recursos para el cuidado de niños y niñas y otras personas dependientes, como por ejemplo guarderías, escuelas de verano, transporte, etc. ${ }^{39}$ En todo caso, será también función del asesor jurídico controlar a un tiempo que tales medidas no perjudiquen el desarrollo profesional.

38 PousADA, T.H.: «Género y cooperativas. La participación femenina desde un enfoque de género», Partes 1 y 2, Cuadernos de trabajo Núms. 18 y 19, Ediciones del Instituto Movilizador de Fondos Cooperativos, Buenos Aires, 2003, pág. 24-25. Recuperado en http://www.centrocultural.coop/modules/wfdownloads/singlefile. php?cid=3\&lid=49, el 9 de mayo de 2011. Citado por Senent, M.J: ob. cit., (nota al pie 31), pág. 78.

39 Vid. Senent, M.J: ob. cit., (nota al pie 32), pág. 78. 
En otro orden, y a fin de visibilizar todas aquellas situaciones que son desfavorables para las mujeres como grupo social, se deben realizar y difundir investigaciones con perspectiva de género. Por consiguiente, las CNA están requeridas de una «Comisión de Educación, Capacitación e Investigación» que materialice este propósito. La función del asesor jurídico en este sentido, es diagnosticar y ejecutar la institucionalización de esta Comisión, superando la omisión de la legislación cooperativa general sobre la materia educativa.

En el propósito de contribuir a la educación cooperativa con perspectiva de género, podría también influir la intercooperación ${ }^{40}$ entre cooperativas; sin embargo, es este un terreno poco explorado en Cuba, tanto desde el punto de vista teórico como en su implementación legal y práctica.

Pese a esta limitación, una mirada aguda al ordenamiento jurídico cubano desde la asesoría jurídica puede ofrecer los fundamentos necesarios para guiar los procesos de intercooperación que reclama la equidad de género en las CNA ${ }^{41}$. Además, estos fundamentos jurídicos podrían enriquecerse mediante el ejercicio de la autonomía regulatoria de la cooperativa, concretándose en sus Estatutos y en otros instrumentos diferentes a los tradicionales contratos con contenido patrimonial y ánimo de lucro. En este caso, los asesores jurídicos pueden aconsejar la formalización de Convenios de Colaboración entre Cooperativas o de estas con otros entes de la sociedad civil como la Federación de Mujeres Cubanas (FMC), a fin de concretar puntos de encuentro o redes de intercambio de mujeres, que fortalezcan la defensa de sus derechos.

40 Muchas de las dificultades que hoy enfrentan las cooperativas en Cuba pudieran paliarse potenciándose la intercooperación, entendida como «la relación voluntaria entre cooperativas, con el propósito de fortalecer la gestión cooperada para el desarrollo en diversos órdenes, a través de procesos planificados y de promoción permanente de la eficiencia productiva, la capacitación, la equidad de género, el cuidado del medio ambiente y el desarrollo territorial, en articulación con otros actores, sin que se afecte la autonomía de las cooperativas que intercooperan». ASOCIACIÓN NACIONAL DE ECONOMISTAS Y CONTADORES DE CUBA (ANEC): Informe de evaluación intermedia externa, Proyecto «Gestión cooperativa en el sector agropecuario en Cuba» (APOCOOP), La Habana, 2017.

41 Ante la falta de regulación específica sobre este aspecto en el ordenamiento jurídico cubano, se recomienda mirar a la Ley No. 59 de 1987, "Código Civil Cubano», ARTíCULO 47. Las causas que generan la relación jurídica son: b) los actos jurídicos. También complementa en este sentido la Ley No. 50 de 1985, "De las Notarías Estatales», ARTíCULO 49.1. El acto jurídico es una manifestación lícita, de voluntad, expresa o tácita, que produce los efectos dispuestos por la ley, consistentes en la constitución, modificación o extinción de una relación jurídica; y ARTíCULO 13. Los documentos públicos que redacta y autoriza el Notario son los siguientes: a) Las escrituras, cuyo contenido es un acto jurídico. 
En esta misma dirección, es oportuno insistir en que la perspectiva de género debe alcanzar al entorno de las mujeres cooperativistas, mediante la conciliación con sus familiares y con sus espacios domésticos en general. Esta idea propone un acercamiento a lo que Atfonso AlEMÁN refiere como Responsabilidad Social Directa, diferente a la que las empresas realizan a través de la intermediación del Estado (indirecta), que presupone «el compromiso consciente de sus directivos y trabajadores con la satisfacción de las necesidades y expectativas de su recurso humano y la comunidad en que se inserta, a partir de una gestión propia y de sus recursos, mediante el vínculo estable y sistemático con las organizaciones políticas, de masas y sociales de su ámbito interno y de su entorno inmediato» ${ }^{42}$. El propio autor enfatiza, acertadamente, que esta Responsabilidad Social Directa es consustancial a la cooperativa, para cuya materialización requiere del compromiso consciente; la participación real; una formación ética e ideológica basada en los valores que la identifican; así como utilidades que sustenten financieramente los diferentes proyectos sociales que desarrolla la entidad; y el reconocimiento y apoyo estatal a la acción social directa que deberá manifestarse, entre otros factores, en la adopción de instrumentos legales que despojen el carácter opcional de la gestión social cooperativa ${ }^{43}$.

Mientras esta dimensión vinculante de la Responsabilidad Social se concreta en la legislación general, los asesores jurídicos pueden aprovechar el «Modelo de Gestión de la Responsabilidad Social Cooperativa Directa» 44 , promovido en su momento por el Centro de Estudios de Desarrollo Cooperativo y Comunitario (CEDECOM) de la Universidad de Pinar del Río, para orientar su implementación a través de las normas jurídicas internas de la CNA. Si bien esta acción no encontrará amparo en el ordenamiento jurídico cubano, tampoco conspiraría ni contra su preceptiva ni contra su esencia popular.

Para ello se cuenta con la definición de sus componentes principales, con potencial para ser traspasados — de principio a fin - por la equidad de género, a saber: a) el «Mercado Social Objetivo», referido al conjunto de necesidades y expectativas presentes en los ámbitos de

42 Alfonso, A.L.: Modelo de gestión de la responsabilidad social cooperativa directa. Estudio de caso: cooperativa de producción agropecuaria Camilo Cienfuegos, Tesis presentada en opción al grado científico de Doctor en Ciencias Económicas, Centro de Estudios de Desarrollo Cooperativo y Comunitario, Facultad de Ciencias Económicas, Universidad de Pinar del Río, Pinar del Río, 2008, pág. 48.

43 Vid. Ibidem, pág. 106-108.

44 Idem. 
actuación (interno y externo); b) el «Sistema de Indicadores Sociales» diseñados a partir de la operacionalización de los principios cooperativos y concretándose en aspectos para medir la gestión de la responsabilidad social que no desconocen la perspectiva de género; y c) el «Balance Social Cooperativo» como una herramienta de información metódica que permite evaluar el nivel de cumplimiento de las actividades que desarrolla la cooperativa para el cumplimiento de su misión social, en un período dado y que debe ser auditable ${ }^{45}$.

Sobre esta base se puede afirmar, que las funciones de diagnóstico, consejería, ejecución y control que comúnmente realiza el asesor jurídico, pueden contribuir a la equidad de género. Puede resultar decisivo en este sentido que se intencione en cada CNA un «plan de igualdad ${ }^{46}$ », compuesto por un conjunto ordenado de medidas adoptadas - previa realización de un diagnóstico - destinadas a alcanzar la igualdad de trato y de oportunidades entre mujeres y hombres, así como para reducir al máximo la discriminación por razón de género ${ }^{47}$.

\section{Conclusiones}

1. La incorporación de una correcta perspectiva de género en las estructuras sociales e institucionales contribuye a su transfor-

45 También se define el Balance Social Cooperativo como «una herramienta de la gestión socioeconómica que facilita a las cooperativas medirse y rendir cuentas, a los/ as asociados/as —sus dueños/as- especialmente, y a todos los demás grupos de interés que son impactados por su accionar en relación con el cumplimiento de su esencia o identidad, es decir, desde los valores y los principios cooperativos. Además de permitir el análisis interno de cada cooperativa, este caudal de información ayuda a construir la imagen del movimiento cooperativo sobre parámetros objetivos y fiables». ACl-AMéRICAS: Balance Social Cooperativo: certificación de Cooperativas de las Américas en Responsabilidad Social Cooperativa, s/f. Recuperado de www.aciamericas.coop.com, el 15 de junio de 2016.

46 Para COCETA, en su Guía para la elaboración de Planes de Igualdad en las Cooperativas de Trabajo, este Plan de Igualdad debe ser universal, para que incida en todas las personas que se integran en la cooperativa y no sólo en las mujeres; transversal, para que implique a todas las áreas de gestión de la organización; dinámico, para que sea progresivo y esté sometido a cambios constantes; sistemático-coherente, pues el cumplimiento de la metodología diseñada llevará a la igualdad real, objetivo final del plan; flexible, pues se diseña a medida de cada entidad y puede ser modificado en función de las necesidades y posibilidades reales; y determinado, pues su duración se limita al momento en que se alcanzan los objetivos propuestos. Vid. COCETA: Guía para la elaboración de planes de igualdad en las cooperativas de trabajo, (s/f). Recuperado de http:// www.coceta.coop/publicaciones/guia-igualdad-cooperativas.pdf

47 Ibidem. 
mación en estructuras equitativas y justas para mujeres y hombres, a través de múltiples beneficios. En el mundo empresarial, las cooperativas son espacios asociativos con rasgos intrínsecos que tienden a la sostenibilidad económica, la democracia, la justicia social y la responsabilidad, condiciones idóneas para lograr la equidad de género. Lamentablemente, la realidad de las CNA en la provincia de Pinar del Río aún dista de alcanzar este ideal, en tanto en estas coexisten una carente perspectiva de género en las normas cooperativas; el bajo nivel de escolaridad de las socias; su escasa representatividad en los cargos de mayor responsabilidad; la sobrecarga laboral de las féminas producto al doble rol de socia-trabajadora y ama de casa; falta de capacitación en temas relativos a la equidad género y pobre articulación de las CNA con la comunidad.

2. La asesoría jurídica es un modo de actuación del profesional del Derecho, a través del cual se desarrolla un proceso de articulación de las funciones de diagnóstico, consejería, ejecución y control al redactar, interpretar y aplicar las normas. El ejercicio de estas funciones para las empresas debe equilibrar los intereses de esta con el interés general expresado en las leyes. Esta labor es trascendental para las cooperativas, en tanto puede contribuir a la consolidación de su identidad. Tal aspiración depende de la implementación del Derecho Cooperativo en los ordenamientos jurídicos, a la vez que de los niveles de especialización de los asesores de cooperativas y de la conciencia crítica-creativa que desplieguen durante el desarrollo de sus funciones.

3. Como resultado de las relaciones de colaboración entre la EPSEL y la UPR, se aspira a perfeccionar, de cara a la identidad cooperativa, la metodología empleada para desarrollar el proceso de asesoría jurídica a las CNA de Pinar del Río. Entre los indicadores hacia los cuales se dirigirán estas propuestas, se halla la equidad de género. En este sentido se propone que la Metodología oriente al asesor jurídico a incidir en la flexibilización de la gestión del tiempo de trabajo y del espacio laboral; en la institucionalización de la educación cooperativa con perspectiva de género; en la formalización de Convenios de Colaboración entre cooperativas o de estas con otros entes, a fin de concretar redes de intercambio en pos de la equidad de género; en la implementación jurídica paulatina de la responsabilidad social directa, contribuyendo a la conciliación de las socias y socios con sus familiares y espacios domésticos en general. 


\section{Bibliografía}

Alianza CoOperativa INTERnacional: Declaración Sobre la Identidad Cooperativa, Manchester, 1995, consultada en EL HOGAR OBRERO: Cooperativa de Consumo, Edificación y Crédito Ltda., en http: //www.elhogarobrero1905. org.ar, consultada el 19 de diciembre de 2009.

- Estrategia para promover la igualdad de género, 2000. Recuperado de http:// www.coop.org/coop/2000-gender-strategy.pdf, el 5 de diciembre de 2018.

- Ley Marco para las Cooperativas de América Latina, Ed. ACI-Américas, San José, 2009.

- Balance Social Cooperativo: certificación de Cooperativas de las Américas en Responsabilidad Social Cooperativa, s/f. Recuperado de www.aciamericas.coop.com, el 15 de junio de 2016.

Alfonso, A.L.: Modelo de gestión de la responsabilidad social cooperativa directa. Estudio de caso: cooperativa de producción agropecuaria Camilo Cienfuegos, Tesis presentada en opción al grado científico de Doctor en Ciencias Económicas, Centro de Estudios de Desarrollo Cooperativo y Comunitario, Facultad de Ciencias Económicas, Universidad de Pinar del Río, Pinar del Río, 2008.

Asociación Nacional de Economistas y CONTAdores de Cuba (ANEC): Informe de evaluación intermedia externa, Proyecto «Gestión cooperativa en el sector agropecuario en Cuba» (APOCOOP), La Habana, 2017.

BRUCH, E.: Marketing de servicios jurídicos a pequeñas y medianas empresas. Universitat Autónoma de Barcelona, 2016, págs. 40-44. Recuperado de https://www.tesisenred.net/bitstream/handle/10803/386530/ebm1 de1.pdf, el 24 de junio de 2018.

CASTRO, R.: Discurso pronunciado por el General de Ejército, Primer Secretario del Comité Central del Partido Comunista de Cuba y Presidente de los Consejos de Estado y de Ministros, en la clausura del IX Período Ordinario de Sesiones de la VIII Legislatura de la Asamblea Nacional del Poder Popular, La Habana, 14 de julio de 2017, Versiones Taquigráficas del Consejo de Estado, Granma, Órgano Oficial del Comité Central del Partido, 15 de julio de 2017.

CEDPA: "Género y Desarrollo: Serie de Manuales de Capacitación de CEDPA». CEDPA Volumen III, 1996. Recuperado de http://www.cedpa.org/images/ pubs-pdfs/gender_spanish_all.pdf el 5 de diciembre de 2018.

Cemborain, E.: Responsabilidad social en las cooperativas: igualdad de oportunidades entre hombres y mujeres, 2006 Recuperado de www.researchgate. net/publication/268299116_Responsabilidad_social_en_las_cooperativas_ igualdad_de_oportunidades_entre_hombres_y_mujeres el 5 de diciembre de 2018.

COCETA: Guía para la elaboración de planes de igualdad en las cooperativas de trabajo, (s/f). Recuperado de http://www.coceta.coop/publicaciones/ guia-igualdad-cooperativas.pdf el 5 de diciembre de 2018.

CracognA, D.: Estudios de Derecho Cooperativo, Intercoop Ed. Cooperativa Ltda., Buenos Aires, 1986. 
Cracogna, D., URiBe, C.: Buen Gobierno Cooperativo. VIII Congreso Nacional Cooperativo, Cartagena de Indias, 2003. Recuperado de http://www.confecoop.coop/images/stories/memorias/2003/dante_uribe\%20_\%28Buen\%20 Gobierno\%, el 5 de diciembre de 2018.

CUADRADO, I.: «Valores y rasgos estereotípicos de género en mujeres líderes». Psicothema, v. 16, n. 2, 2004.

CUBADEBATE: Autorizadas 16 cooperativas no agropecuarias en primer trimestre de 2016, 2016. Recuperado de http://www.cubadebate.cu/ noticias/2016/06/29/autorizadas-16-cooperativas-no-agropecuarias-en-primer-trimestre-de-2016/, el 4 de octubre de 2016.

CuBAdebATE: Autorizadas 16 cooperativas no agropecuarias en primer trimestre de 2016, 2016. Recuperado de http://www.cubadebate.cu/ noticias/2016/06/29/autorizadas-16-cooperativas-no-agropecuarias-en-primer-trimestre-de-2016/, el 4 de octubre de 2016.

Esteban, M.L., Gargallo, A. \& Pérez, F.J.: « ¿Presentan las cooperativas contextos favorables para la igualdad de género? Especial referencia a la provincia de Teruel». CIRIEC-España, Revista de Economía Pública, Social y Cooperativa, 88, 2016.

García, A.: Derecho cooperativo y de la Economía Social y Solidaria, Asociación Iberoamericana de Derecho Cooperativo, Mutual y de la Economía Social y Solidaria, Mérida, 2017.

HENRŸ, H.: Orientaciones para la legislación cooperativa, OIT, Ginebra, 2013.

HERNÁNDEZ, O.: "La relación género y cooperativa en el ordenamiento jurídico cubano. Una aproximación a su realidad y a sus perspectivas ante el proceso de perfeccionamiento del modelo económico en el país». Boletín de la Asociación Internacional de Derecho Cooperativo, Núm. 46, 2012.

- «La relación género y cooperativa en Cuba. Perspectivas dentro del marco jurídico experimental de la cooperativa». Revista de Estudios Cooperativos, N. 2, 2013.

- «Las cooperativas no agropecuarias en Pinar del Río. Una aproximación al enfoque de género en su funcionamiento». Revista de Estudios Cooperativos. Núm. 6, 2015.

- «La relación entre enfoque de género y trabajo en las Cooperativas No Agropecuarias en Pinar del Río. Reflexiones para su perfeccionamiento.» Ponencia presentada en $X$ Encuentro de Investigadores Latinoamericanos en Cooperativismo, Buenos Aires, Argentina, 2018.

Hernández, O; VAlle, D.: La Cooperativa No Agropecuaria Atelier «La Moda»: Aproximación a la experiencia de una cooperativa de mujeres En Rodríguez, O., Hernández, O. (Compiladores): «Apuntes de Derecho Cooperativo para Cuba». Ediciones Loynaz, Pinar del Río, 2018.

La transformación de la función jurídica. Los siete retos de las asesorías jurídicas internas, s/f. Recuperado de https://www.pwc.es/es/publicaciones/legal-fiscal/ assets/informe-pwc-transformacion-funcion-juridica.pdf, el 24 de junio de 2018.

Lineamientos frente al Código de Buen Gobierno para el Sector SolidarioCooperativas. Manual Guía Gobierno Corporativo Cooperativas, EMPREN- 
DER, 2017. Recuperado de https://www.google.com/url?sa=t\&rct=j\&q=\& esrc $=$ s\&source $=$ web $\& c d=2 \& v e d=2$ ahUKEwjE-O_NIKbfAhXNmVkKHS9xC5 gQFjABegQIARAC\&url=http \%3A\%2F\%2Fwww.campusvirtualemprender. com\%2Fmoodle\%2Fpluginfile.php\%2F2555\%2Fblock_html\%2Fcontent \%2FManual\%2520Gui\%25CC\%2581a\%2520Gobierno\%2520Corporati vo\%2520Cooperativas.pdf\&usg=AOvVaw0jpF7d2yVeKqQbxO4_jJ6O, el 5 de diciembre de 2018.

MénDEZ. N.: «Estrategia de impulso al modelo cooperativo entre mujeres». Comité Regional de Equidad de Género (CREG), ACI Américas, 2011. Recuperado de www.aciamericas.coop/IMG/pdf/estrategiamodelocoopmujeres. pdf, el 5 de diciembre de 2018.

Ministerio de FinANZAS y PRECIOS: Aprobada extinción de Cooperativa de servicios contables SCENIUS, 2017. Recuperado en http://www.cubadebate.cu/ noticias/2017/08/07/aprobada-extincion-de-cooperativa-de-servicios-contables-scenius/\#.Wb54j3uR7mM, el 12 de septiembre de 2017.

MonteCino. S.: «Palabra Dicha. Escritos sobre género, identidades, mestizajes». Universidad de Chile, Facultad de Ciencias Sociales, 1997. Recuperado de www.uchile.cl/documentos/palabra-dicha-escritos-sobre-genero-identidades-mestizajes-pdf-9839kb_56703_8.pdf el 5 de diciembre de 2018.

Oficina InTERnACIONAL DEL Trabajo: Guía para la incorporación de la perspectiva de género en las estrategias de desarrollo económico local. Programa de DesarroIlo Económico Local, Oficina para la Igualdad de Género, OIT, Ginebra 2010.

ORganizACIÓn INTERNACIONAL DEL TRABAJO: Política de la OIT sobre la incorporación de la perspectiva de género, 1999. Recuperado de http://www.ilo.org/public/spanish/ bureau/gender/newsite2002/about/defin.htm el 5 de diciembre de 2018.

PIÑEIRO, C.: Cooperativas y Socialismo. Una mirada desde Cuba. Editorial Camino, La Habana, 2012.

PIÑEIRO. C.: Diagnóstico preliminar de las cooperativas no agropecuarias en La Habana, Cuba, 2014. Recuperado de https://www.ceec.uh.cu/file/569/ download?token=VfqEKMZE el 18 de abril de 2016.

PUIG, Y.: Autoridades explican nuevas medidas respecto a cooperativas no agropecuarias, 2017. Recuperado de http://www.cubadebate.cu/ noticias/2017/08/09/autoridades-explican-nuevas-medidas-respecto-a-cooperativas-no-agropecuarias/, el 12 de septiembre de 2017.

RODRíGUEZ, O.: La constitucionalización de la cooperativa. Una propuesta para su redimensionamiento en Cuba. Ed. Vincere Asociados, Coletânea IBECOOP (No. 1), Brasilia-DF, 2017.

Salinas, A.: Derecho Cooperativo, Ed. Cooperativismo, México, 1954.

SENENT, M.: ¿Cómo pueden aprovechar las cooperativas el talento de las mujeres? Responsabilidad Social Empresarial e Igualdad Real, REVESCO N. ${ }^{\circ} 105$ - Monográfico: La economía social y la igualdad de género, 2011.

SERNAM, ACCIÓN RSE: Guía para impulsar la Equidad de Género en las empresas, 2006. Recuperado de http://www.centroscomunitariosdeaprendizaje. org.mx/sites/default/files/guia_para_impulsar_la_equidad.pdf, el 5 de diciembre de 2018. 
TRISTÁ, G.: Cooperativas no Agropecuarias, conferencia ofrecida y reproducida a militantes del Partido Comunista de Cuba por la Comisión de Implementación de los Lineamientos del Partido y la Revolución, 2015.

Vasallo, N., Almeida, Y., Rivera Y., \& Romero, M.: Género: Aspectos conceptuales y su aplicación. Publicaciones Acuario, La Habana, 2011.

VASALLO, N.: El género: Un análisis de la «naturalización» de las desigualdades. Editorial CEDEM, La Habana, 2004.

VIRGILI. D.: "Género y trabajo remunerado. Experiencia grupal de investigaciónacción con mujeres y hombres dirigentes del Ministerio de Transporte». Tesis en opción al título de Máster en Psicología Educativa. Universidad de la Habana. Facultad de Psicología, 2012.

\section{Legislación}

Decreto Ley 349 de 24 de enero de 2018, «Del Asesoramiento Jurídico», Gaceta Oficial No. 5 Extraordinaria de 24 de enero de 2018.

Resolución 41 de 3 de marzo de 2018 del MINJUS «Reglamento para el ejercicio de la actividad de asesoramiento jurídico», Gaceta Oficial No. 20 Extraordinaria de 5 de marzo de 2018.

Ley No. 59 de 16 de junio de 1987, «Código Civil» cubano, Gaceta Oficial Extraordinaria No. 9, del 15 de octubre de 1987.

Ley No. 50 de 1985, "De las Notarías Estatales», Ministerio de Justicia, La Habana, 1989. 